\title{
Evolución de la siniestralidad de personas que se transportan en moto en la ciudad de Medellín, Colombia en el periodo 2008-2014
}

\author{
Road traffic injury evolution of motorcycles riders in Medellin, Colombia during the period 2008-2014
}

Luis Fernando Restrepo-Betancur1, Joaquín Angulo-Arizala²

1 Estadístico, Especialista en Estadística, Especialista en Biomatemática. Profesor Titular Facultad de Ciencias Agrarias, Universidad de Antioquia, Grupo Giser. Medellín, Colombia. e-mail: frbstatistical@yahoo.es

2 Zootecnista, Esp, MSc, PhD en Ciencias Animales. Profesor Asociado, Universidad de Antioquia, Grupo Grica. Medellín, Colombia. e-mail: joaquinangulo@gmail.com

\begin{abstract}
Resumen
Introducción: Los siniestros de tránsito son considerados un problema de salud pública a nivel mundial que repercuten en la sociedad, e inciden en la economía y en el sistema de salud de un país. Se considera dentro de las principales causas de muerte en el mundo. Entre los siniestros de tránsito, los asociados con las motos han llamado la atención a investigadores, debido a su alta participación porcentual. Objetivo: Evaluar los siniestros de moto ocurridos en la ciudad de Medellín-Colombia entre los años 2008 al 2014. Materiales y métodos: Se tomó la información reportada mensualmente por la Secretaría de Tránsito de 107.125 casos relacionados con los siniestros de tránsito asociados con los conductores de moto. Se utilizó análisis probabilístico, descriptivo exploratorio de tipo unidimensional y análisis MANOVA. Resultados: Se encontró un incremento significativo en los siniestros asociados con la conducción de motos, en la ciudad de Medellín en los últimos años, detectando diferencia $(\mathrm{p}<0,01)$ entre sexos, entre rangos de edad y tipos de siniestro. Conclusión: Esta investigación permitió evidenciar una tendencia creciente en la cantidad de heridos y personas fenecidas asociadas a la participación de motociclistas o de acompañantes, anotando que la mayoría pertenecen al sexo masculino, en edades que oscilan entre los 20 y 29 años.
\end{abstract}

Palabras clave: Accidentes de tránsito; probabilidad; salud pública. (Fuente: DeCS, Bireme).

\begin{abstract}
Introduction: Road traffic injuries are considered a public health problem worldwide which have an impact on society, economy and the health system of a country. It is considered one of the main causes of death around the world. Among road traffic injuries, those associated with motorcycles have drawn attention to researchers due to its high percentage participation. Objective: To evaluate motorcycle accidents that occurred between 2008 and 2014 in Medellin, Colombia. Materials and methods: The information reported monthly by the Secretariat of Transportation of 107.125 cases related to traffic accidents associated with motorcycle riders was taken. Probabilistic analysis, descriptive and exploratory analysis of dimensional type, and MANOVA were used. Results: A significant increase in road traffic injuries associated with motorcycle riding was found in the city of Medellin during the last years as well as the difference $(\mathrm{p}<0,01)$ between sexes, age ranges and types of loss. Conclusion: This research allowed to highlight
\end{abstract}


an increasing trend in the number of wounded and defunct people associated with the participation of motorcycle riders or passengers, noting that the majority of them were males between 20 and 29 years old.

Keywords: Road traffic accidents; probability; public health. (Source: DeCS, Bireme).

\section{Introducción}

Los traumatismos asociados con los siniestros de tránsito conforman un problema de salud pública clasificándose como una epidemia silenciosa que repercute en todos los sectores de la sociedad representando uno de los principales factores que incide en la morbilidad y en el fallecimiento de personas a nivel orbital.1,2 La inferencia estadística ha permitido pronosticar que cada año, en el mundo mueran 1,2 millones de personas debido a factores asociados con la movilidad en las vías públicas, donde los conductores de moto representan un alto porcentaje en los siniestros y en el número de heridos.3 En los países de economía menos favorecida se presentan los mayores porcentajes de defunciones de motociclistas, anotando que estos cuentan con menos de la mitad del parque vehicular mundial.4-6 En los países que poseen un ingreso per cápita alto, se ha detectado que la estadística asociada a la tasa de mortalidad ha ido descendiendo en los últimos veinticinco años, de acuerdo con investigaciones adelantadas y relacionadas con el tema.4

La importancia sanitaria de los siniestros asociados con los motociclistas está fundamentada en una alta morbilidad y mortalidad que se desprenden de dicho suceso. Presentando politraumatismos de índole físico y traumas psíquicos que se producen en los pacientes que han sido afectados por hechos sucedidos en las vías, incidiendo en el estado emocional de las personas. Lo anterior repercute en altos costos económicos que se derivan de la inactividad y recuperación del afectado.7

En ciertos países se ha detectado que mientras menor sea el nivel socioeconómico de una persona, mayor es el riesgo de sufrir un traumatismo vinculado con una colisión de motos o de otros medios de transporte utilizados, lo que está denotando una relación inversa; identificando a la pobreza como un factor de riesgo importante. ${ }_{8,9}$ En los estados de ingreso per cápita bajo y medio, las pérdidas económicas como consecuencia directa de la muerte o discapacidad asociadas con los traumatismos causados por el tránsito, pueden significar alrededor del $2 \%$ de su producto interno bruto.4 Se ha reportado que los gastos pueden ascender al 5\% del PIB, como se presentó en los países de Sudáfrica y de Malawi en años anteriores.10 Los traumatismos correlacionados con acontecimientos de tránsito representan el principal factor de muerte en personas cuya edad oscila entre los (10-24) años a nivel mundial y constituyen alrededor del $15 \%$ de todas las muertes en varones.11,12 Según la Organización Mundial de la Salud, el costo global como consecuencia de choques y las consecuentes asociadas con las lesiones o muertes causadas por siniestros de tránsito en el 2004, fue de 518.000 millones de dólares.2

En Colombia en el año 2008 los siniestros de tránsito fueron la segunda causa de muerte violenta después de los homicidios, donde las personas del sexo masculino tienen mayor participación respecto al sexo femenino. El 51,7\% de las personas heridas fueron motociclistas, anotando que los ocupantes de motocicleta en su mayoría eran personas jóvenes.13 En el año antes citado el Instituto Nacional de Medicina Legal y Ciencias Forenses de Colombia, registró dentro de sus estadísticas 5.290 muertes y 40.377 lesionados por causas derivadas de manera directa con los eventos asociados a la movilidad.14

En la ciudad de Medellín en el año 2010 se encontró que el $62,3 \%$ de los casos de siniestralidad se asociaron con conductores de moto.15 Lo que ratificó las estadísticas detectadas en otra investigación adelantada en la misma ciudad, la cual permitió detectar un alto crecimiento en la participación de las motos en la accidentalidad ocurrida, lo que generó un significativo incremento en la mortalidad y en el 
número de heridos como consecuencia del choque producido por dicho medio de transporte. Destacándose las siguientes cifras: el 29,6\% de los motociclistas presentaron traumas de tejido blando, el 20,6\% traumas en las extremidades inferiores, el 30\% trauma de cráneo.16

Esta investigación tuvo como objetivo, evaluar los siniestros de moto ocurridos en la ciudad de Medellín - Colombia, con base en la información reportada por la Secretaria de Tránsito.

\section{Materiales y métodos}

Se realizó un estudio descriptivo retrospectivo, basado en fuentes secundarias de información reportadas por la Secretaría de Transporte de la ciudad de Medellín Colombia en el periodo comprendido entre los años 2008 al 2014. La información relacionada con los siniestros de tránsito asociados con la conducción de motos, se tomó mensualmente, de acuerdo con los reportes consignados en la base de datos de dicha entidad. La población objeto (107.125 casos) estuvo relacionada con todos los motociclistas fallecidos o heridos en el lugar del siniestro para el periodo citado, discriminado por sexo, rango de edad y tipo de accidente. Se excluyó a los que fallecieron hasta 30 días después. Las variables evaluadas se reportan en la tabla 1.

Tabla 1. Variables evaluadas y modelos asociados

\begin{tabular}{lll}
\hline $\begin{array}{l}\text { Variable de } \\
\text { control }\end{array}$ & $\begin{array}{l}\text { Tipo de } \\
\text { variable }\end{array}$ & $\begin{array}{l}\text { Distribución } \\
\text { probabilística }\end{array}$ \\
\hline Sexo & Categórica & Binomial \\
Tipo de accidente & Categórica & Multinomial \\
Rango de edad & Categórica & Multinomial \\
$\begin{array}{l}\text { Variables } \\
\text { respuesta }\end{array}$ & $\begin{array}{l}\text { Tipo de } \\
\text { variable }\end{array}$ & $\begin{array}{l}\text { Distribución } \\
\text { probabilística }\end{array}$ \\
$\begin{array}{l}\text { Siniestros fatales } \\
\text { moto }\end{array}$ & $\begin{array}{l}\text { Cuantitativa } \\
\text { discreta }\end{array}$ & Poisson \\
Heridos moto & $\begin{array}{l}\text { Cuantitativa } \\
\text { discreta }\end{array}$ & Poisson \\
\hline
\end{tabular}

Para controlar un posible sesgo de información, teniendo en cuenta la base de datos reportada por la Secretaría de Tránsito, se efectuó un estricto control de calidad en las variables de interés digitadas en la nueva base, a fin de poder garantizar la idoneidad de los datos. Infortunadamente los reportes efectuados por parte del ente citado adolece de factores específicos que pudieran haber servido para un análisis más detallado en relación a la causa concreta de la muerte o la herida de las personas que transitaban en moto.

Para el abordaje estadístico de los datos, se empleó análisis multivariado de la varianza MANOVA con contraste canónico de tipo ortogonal, determinando vía máxima verosimilitud la dimensionalidad del contraste, anotando que se utilizó la familia Box-Cox a fin de transformar la información y así garantizar los supuestos asociados con el modelo. El análisis se complementó con distribuciones de frecuencia de tipo univariado y con cálculos probabilísticos de interés de acuerdo a la naturaleza de la variable evaluada. Finalmente, se realizó análisis descriptivo y de correlación por el método de Spearman. Se empleó un nivel de significancia del $5 \%$ en las inferencias efectuadas. Se utilizó el paquete estadístico SAS University.

\section{Consideraciones éticas}

Por tratarse de un estudio de tipo retrospectivo, basado en fuentes secundarias, se clasificó en la categoría sin riesgo según la Resolución 8340 de 1993 del Ministerio de Salud de Colombia. Anotando que se tomaron únicamente los datos reportados por el ente público (Secretaría de Transporte de Medellín).

\section{Resultados}

En la tabla 2 se describe la dinámica de comportamiento asociada con los siniestros de moto los cuales están discriminados por tipo. Al observar el coeficiente de variación, se aprecia la mayor homogeneidad en la variable: número de muertes $(5,3 \%)$ en el periodo de tiempo evaluado. La variable con la característica más heterogénea fue el número de heridos moto, con un coeficiente de variación del 22,9\%. En los años (2011, 2012, 2013 y 2014) las muertes de tránsito asociadas con la moto representaron más del $40 \%$ de los casos, alcanzando el máximo porcentaje en el año 
2012 con el 47,2\%. En lo referente al número de heridos derivados de la conducción de motos, este va en aumento, para el año 2008 el porcentaje detectado fue del 54,0\% y en el año 2014 fue del $71,9 \%$. No se detectó una relación entre el número de muertos y el número de heridos asociados con la conducción de la moto $(\mathrm{p}=0,148)$. Se detectó diferencia entre los años $(2011,2012$, 2013 y 2014) con respecto a los años $(2008,2009$ y 2010) en lo relacionado al número de personas siniestradas en moto.
En el gráfico 1 se observa en primer lugar, una marcada diferencia en el número de personas muertas pertenecientes al sexo masculino con respecto al femenino asociadas a la conducción de motos, notándose un ascenso marcado en el año 2011 que se mantiene relativamente contante hasta el 2013, año a partir del cual empieza a descender; y en segundo lugar, para el caso del sexo femenino, que la tendencia también es ascendente y un poco más marcada a partir del año 2012.

Tabla 2. Número de eventualidades por año relacionadas con la conducción de moto, con respecto al total. Medellín Colombia, 2008 al 2014

\begin{tabular}{|c|c|c|c|c|c|c|}
\hline Año & Total muertos & Muertos moto & $\%$ & $\begin{array}{c}\text { Total } \\
\text { Heridos }\end{array}$ & Heridos moto & $\%$ \\
\hline 2008 & 326 & 117 & 35,9 & 18178 & 9826 & 54,0 \\
\hline 2009 & 301 & 108 & 35,8 & 21148 & 12628 & 59,7 \\
\hline 2010 & 293 & 88 & 30,0 & 21318 & 13284 & 62,3 \\
\hline 2011 & 307 & 133 & 43,3 & 23835 & 15535 & 65,2 \\
\hline 2012 & 275 & 130 & 47,2 & 24870 & 16815 & 67,6 \\
\hline 2013 & 306 & 134 & 43,7 & $26826^{*}$ & $18778^{*}$ & $70,0^{*}$ \\
\hline 2014 & 289 & 125 & 43,2 & $27015^{*}$ & $19424^{*}$ & $71,9 *$ \\
\hline CV\% & 5,3 & 13,9 & & 13,9 & 22,9 & \\
\hline Correlación & \multicolumn{4}{|c|}{ Muertes moto y heridos moto } & 0,60 & $p=0,148$ \\
\hline
\end{tabular}

Gráfico 1. Dinámica de fallecidos en accidente de moto por sexo

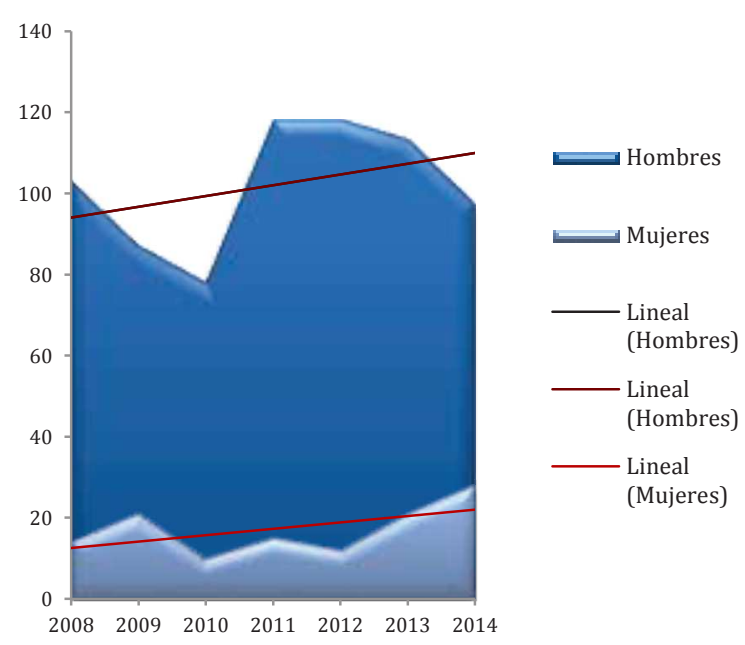

Fuente: Elaboración propia con base en los datos reportados por la Secretaría de Transporte de la ciudad de Medellín Colombia años 2008-2014
Existe un 0,82 de probabilidad de que al ocurrir un siniestro de tránsito con víctimas fatales en moto, la persona que fallezca pertenezca al sexo masculino y para el sexo femenino la probabilidad es de un 0,18. El rango de edad donde se presenta la mayor probabilidad de muerte cuando ocurre un siniestro en moto, oscila en el intervalo comprendido entre los 20 y 29 años. La causa más probable de muerte en moto es por choque con una probabilidad del 0,66 seguida de la caída del conductor o del parrillero con 0,22 como se aprecia en la tabla 3.

Existe un 0,58 de probabilidad que al suceder un siniestro de tránsito resulte herido un motociclista o su acompañante. Al acontecer un hecho fortuito de tránsito en moto es más probable que resulte herido un hombre, que una mujer. La edad donde se presentó la mayor probabilidad está en el rango entre los 20 a 29 años. (Tabla 4). 
Tabla 3. Carta probabilística de muertes asociadas con siniestro de tránsito en moto

\begin{tabular}{lc}
\hline Por tipo & Probabilidad \\
\hline Motociclista o acompañante & 0,491 \\
Por sexo & \\
Hombre & 0,820 \\
Mujer & 0,180 \\
Por rango de edad & \\
De 0 a 9 años & 0,009 \\
De 10 a 19 años & 0,094 \\
De 20 a 29 años & 0,398 \\
De 30 a 39 años & 0,268 \\
De 40 a 49 años & 0,194 \\
Más de 50 años & 0,037 \\
Peatón & \\
Peatón muerto por moto & 0,482 \\
Por tipo de accidente & \\
Choque & 0,665 \\
Caída & 0,225 \\
Atropello & 0,022 \\
Volcamiento & 0,014 \\
Otro & 0,074 \\
\hline Fuente: Datos estimados por los investigadores a partir del reporte de la \\
Secretaría de Transporte. Medellín - Colombia 2008-2014
\end{tabular}

Tabla 4. Carta probabilística de heridos asociados con siniestro de tránsito en moto

\begin{tabular}{lc}
\hline Por tipo & Probabilidad \\
\hline $\begin{array}{l}\text { Motociclista o acompañante } \\
\text { Por sexo }\end{array}$ & 0,580 \\
Masculino & 0,715 \\
Femenino & 0,285 \\
Por rango de edad & \\
De 0 a 9 años & 0,005 \\
De 10 a 19 años & 0,102 \\
De 20 a 29 años & 0,484 \\
De 30 a 39 años & 0,296 \\
De 40 a 49 años & 0,082 \\
> de 50 años & 0,031 \\
Peatón & \\
Peatón herido por moto & 0,615 \\
Por tipo de accidente & \\
Choque & 0,558 \\
Caída & 0,318 \\
Atropello & 0,044 \\
Volcamiento & 0,026 \\
Otro & 0,054 \\
Fuente: Probabilidad calculada por los investigadores a partir del reporte de \\
la Secretaría de Transporte de la ciudad de Medellín-Colombia. 2008-2014
\end{tabular}

Al efectuar el análisis multivariado de la varianza por medio del modelo lineal general (GLM), se detectó diferencia estadística entre sexos ( $p=0,0019)$ relacionado con el número de heridos asociados con la conducción de moto en la ciudad de Medellín - Colombia para el periodo comprendido entre los años 2008 al 2014. De igual manera, se detectaron diferencias entre sexos para el número de personas muertas por la conducción antes citada ( $\mathrm{p}=0,0013)$, así como diferencias estadísticas, tanto para heridos, como fenecidos en hechos relacionados con el transporte en moto, según rango de edad y tipo de accidente. Anotando que las variables dependientes se asociaron con la cantidad de hechos detectados para diferentes condiciones de observación. (Tabla 5).

Tabla 5. Análisis multivariado de la varianza

\begin{tabular}{lcrr}
\hline $\begin{array}{l}\text { Prueba } \\
\text { estadística }\end{array}$ & \multicolumn{1}{l}{$\begin{array}{l}\text { Sexo } \\
\text { Valor } \mathbf{p}\end{array}$} & $\begin{array}{l}\text { Por tipo } \\
\text { Valor } \mathbf{p}\end{array}$ & $\begin{array}{l}\text { Rangos edad } \\
\text { Valor } \mathbf{p}\end{array}$ \\
\hline Heridos (moto) & & & \\
Wilks' Lambda & 0,0019 & 0,0012 & 0,0031 \\
Pillai's Trace & 0,0019 & 0,0012 & 0,0031 \\
Hotelling-Lawley & 0,0019 & 0,0012 & 0,0031 \\
Roy's Greatest R & 0,0019 & 0,0012 & 0,0031 \\
Siniestros fatales (moto) & & \\
Wilks' Lambda & 0,0013 & 0,0017 & 0,0215 \\
Pillai's Trace & 0,0013 & 0,0017 & 0,0215 \\
Hotelling-Lawley & 0,0013 & 0,0017 & 0,0215 \\
Roy's Greatest R & 0,0013 & 0,0017 & 0,0215 \\
\hline
\end{tabular}

En el gráfico 2, se aprecia que la muerte de conductores de moto o de su acompañante se clasifica en el mismo grupo que los peatones muertos por siniestros fatales de tránsito. Las personas entre 20 a 29 años de edad que fenecieron conduciendo moto están en un clúster, en otro grupo se clasificaron los extremos de edad de 0 a 9 años y los mayores a 50 años.

\section{Gráfico 2. Análisis clúster muertes de motociclistas por rango de edad y tipo general Análisis clúster muertes por tipo}

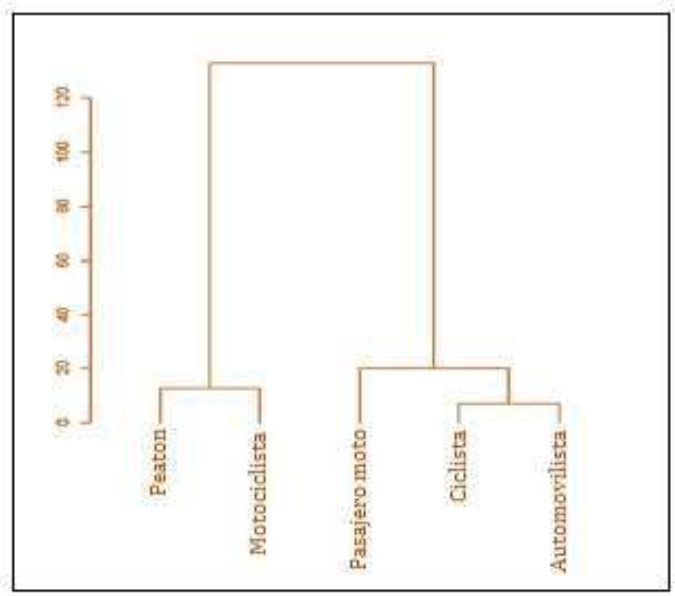


Análisis clúster muertes por rango de edad (moto)

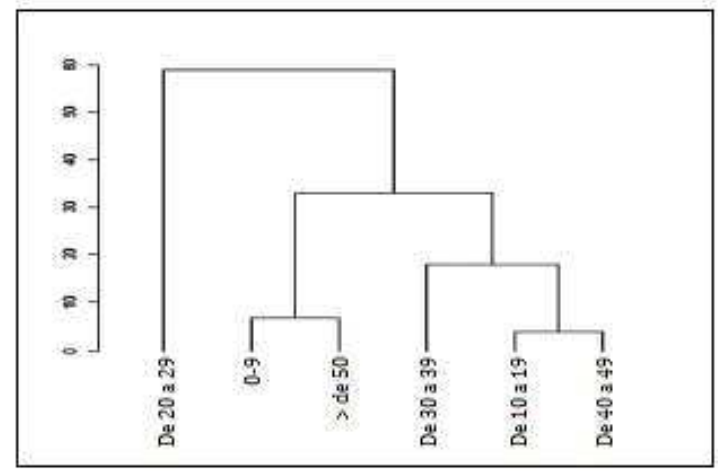

\section{Discusión}

En Medellín en los últimos años se ha hecho una notable inversión en capital económico y en personal humano, con el fin de efectuar una capacitación acertada a conductores de moto, vehículos y peatones. Adicionalmente, se ha implementado una legislación que tiene como objetivo garantizar la seguridad vial en las vías minimizando el riesgo en las mismas. De otro lado, los avances tecnológicos por medio de la instalación de cámaras de última generación, han permitido observar una disminución en el número de siniestros en los lugares donde se ubican estos medios. No obstante en el presente estudio se describe un crecimiento en la participación de las motos en la siniestralidad, esto se puede atribuir en gran medida a la forma fácil de adquisición de dichos aparatos, que se entregan en la mayoría de concesionarios sin cuta inicial y con amplias facilidades de pago, lo que resulta bastante llamativo para trabajadores, estudiantes y público en general.

En un estudio adelantado en la ciudad de Medellín, se encontró que la impericia es uno de los principales factores de riesgo, asociados con los siniestros de moto.17 Según el Fondo de Prevención Vial,18 solo el 16\% de los motociclistas realiza el curso de conducción para obtener su licencia de manera legal, mientras que el $84 \%$ restante obtiene su licencia de manera irregular. Afortunadamente, la nueva normativa que rige para aquellas personas que desean tener un pase de conducción para moto, ha aumentado el número de horas en la capacitación para los futuros conductores en aspectos relacionados con la parte mecánica del medio de transporte, en la información sobre las normas de transporte, y en el manejo mismo del vehículo. Se espera que esto repercuta de manera positiva en minimizar la accidentalidad en la ciudad de Medellín.

De acuerdo con el Comité de Ensambladoras de Motocicletas Japonesas, 19 en Colombia se necesita que los entes gubernamentales de transporte representados en el Ministerio, diseñen políticas y directrices enfocadas a la convivencia segura de vehículos, motos y peatones. Así mismo, el estudio de Vásquez indica que las personas consideran que es la comunidad y sobre todo, los entes que la regulan los responsables de señalar las buenas acciones para que los conductores adopten hábitos, conductas y comportamientos adecuados al conducir. Se requiere que el entorno social, legal, cultural, económico y ambiental les dé soporte. 20 La convivencia entre todos los actores que están presentes en un momento dado en la vía, está referido en el informe emitido por la Secretaría de Transportes y Tránsito de Medellín en el año 2010.21

La presente investigación permitió determinar una alta probabilidad de que al ocurrir un siniestro donde esté uno o más motociclistas involucrados, existen consecuencias lamentables, lo cual es entendible si se analiza la alta exposición del cuerpo del conductor o del acompañante que participan en el hecho. En Medellín se ha observado que hay conductores que manejan sin casco, o si lo tienen no lo llevan de manera correcta, lo que incide en incrementar la probabilidad antes citada. Según la OMS el uso de casco reduce traumatismos craneales mortales y graves hasta en $45 \% .22$

Los cascos deben cumplir una norma reconocida de seguridad; también es fundamental que se abroche correctamente para que surtan el máximo efecto, en más del $74 \%$ de los países, la utilización del casco es obligatoria para los conductores y pasajeros de vehículos de motor de dos o tres ruedas, en todas las vías de tránsito, con independencia del cilindraje. Un $43 \%$ de los países no establecen normas de homologación 
para los cascos, sólo el 40\% de los países tienen en vigor leyes sobre la utilización del casco en motocicletas que se aplican, tanto a los conductores como a los pasajeros y que exigen que los dispositivos en cuestión se ajusten a una determinada norma nacional o internacional.23 En América Latina, la mayoría de los países tienen leyes, al menos formuladas, sobre límites de velocidad, conducir en estado de embriaguez, uso de $\operatorname{cascos} .24$

En esta investigación se detectó un crecimiento notable en el número de heridos derivados de eventualidades ocasionadas por hechos en los cuales se ven involucrados conductores y acompañantes de moto. En el año 2014 se duplicó el número de heridos con respecto al año 2008; en cuanto a las personas fallecidas y reportadas en la base de datos de la Secretaría de Tránsito de la ciudad de Medellín se ha observado una dinámica similar, fluctuando entre 125 y 134 casos, sin diferencia estadística durante los años 2011 al $2014(\mathrm{p}>0,05)$.

En lo relacionado al sexo, existe un 0,82 de probabilidad de que la persona que muera, sea hombre, donde la edad más común de presentación de la víctima oscila entre los 20 a 29 años, los grupos menos afectados están en los extremos de la pirámide de edad. Un estudio realizado en Uruguay permitió mostrar que el $70 \%$ de las personas siniestradas fatalmente eran hombres, con edades fluctuantes entre los 15 a 19 años de edad.25 Cabe anotar que el tipo de accidente de moto más común está asociado con el choque del motociclista, seguido de la caída de éste o de su acompañante.

En cuanto a los hechos relacionados con el número de heridos correlacionados con el manejo de las motos, se encontró que la mayor probabilidad está relacionada con el sexo masculino $(0,71)$, presentándose el mayor número de casos entre los 20 a 29 años con una probabilidad del $(0,48)$, asociados al choque del conductor de moto $(0,55)$. Otro estudio realizado en Medellín entre los años 2004 al 2007, permitió evidenciar que los motociclistas representaron el $38 \%$ de los heridos, en 2005 el $43 \%$ y en el primer semestre de 2006 el 51\%.18 La presente investigación reportó porcentajes superiores al $70 \%$ para el año 2014, en relación al grupo de edad más vulnerable, se encontró divergencia respecto a la investigación realizada en la ciudad de Medellín entre los años 2005 al 2008, en la cual las personas más afectadas eran las mayores de cincuenta años.26

Los siniestros de tráfico causan más víctimas que las guerras, es por este motivo que se debe continuamente diseñar investigaciones que permitan definir estrategias y planes de acción para minimizar el número de víctimas y de personas heridas relacionadas con el tránsito, principalmente las orientadas a motociclistas, que son los principales actores. En la ciudad de Santiago de Cali se ha implementado un sistema de circulación, especialmente diseñado para motociclistas, donde estos cuentan con una vía exclusiva para su movilidad, siendo un sistema pionero a nivel nacional. 27,28

Una investigación adelantada por la Universidad de Oxford permitió mostrar que el aumento de la siniestralidad de los motoristas esta correlacionada con la economía. Ante la presencia de crisis económica los conductores tienden a comprar motos, con el fin de ahorrar, aumentan el número de motocicletas en el sistema, lo que significa, mayor probabilidad de riesgos y por ende más víctimas. 29 Uno de los factores que permite minimizar las muertes en siniestros de moto es el uso adecuado de casco, como se cita en un estudio realizado en Uruguay, el cual debe tener cuatro componentes técnicos de calidad básicos: armazón exterior rígido, relleno que absorba el impacto, espuma interior y sistema de retención.30

\section{Conclusiones}

$\mathrm{Al}$ evaluar los siniestros de moto ocurridos entre los años 2008 al 2014 en la ciudad de Medellín Colombia con base en la información reportada por la Secretaria de Tránsito, se puede concluir que han aumentado de manera notoria en lo relacionado al número de heridos, presentando un incremento de cerca del $50 \%$ entre los años referidos. En cuanto al número de víctimas fatales el incremento ha sido del $7 \%$. 


\section{Recomendaciones}

En la ciudad de Medellín falta sensibilizar más a conductores de motos en relación a las normas existentes de tránsito, con el fin de reducir los siniestros, al igual que a peatones y conductores de otros medios de transporte. Los medios de comunicación juegan un papel vital en relación a la difusión de campañas dirigidas a reducir la velocidad, la ubicación correcta de cascos, el no culebreo, el no adelanto incorrecto de la moto, el respeto al peatón, la revisión técnico mecánica del vehículo de transporte, la necesidad de tener el Soat actualizado, entre múltiples factores.

Se recomienda a la Secretaría de Tránsito de la ciudad de Medellín, crear planes de incentivo para aquellos conductores que presenten un buen manejo vial. De manera adicional dicho ente, en asocio con la Secretaria de Educación están llamados a implementar una cátedra sobre aspectos viales, que se imparta a nivel primario.

Se debe implementar un sistema estadístico más eficiente, mediante la creación de una base de datos nacional única, que permita conocer en detalle todos los factores asociados con el siniestro de moto.

\section{Agradecimientos}

Los autores agradecen al CODI (Comité de Desarrollo de la Investigación), Estrategia de Sostenibilidad 2011-2012 de la Universidad de Antioquia por proporcionar los recursos para este estudio, a la estudiante Elizabeth Ballesteros C por su participación y a la Secretaría de Tránsito de Medellín por facilitar el acceso a los registros.

Conflicto de intereses: Los autores declaran que no existe ningún conflicto de intereses.

\section{Referencias}

1. Organización Panamericana de la Salud, Defunciones por accidentes de tránsito en países seleccionados de las Américas 1985-2001. Boletín epidemiológico. 2004;25(1).

2. Organización Mundial de la Salud, Estrategia quinquenal de la OMS para la prevención de las lesiones por accidentes de tráfico, Accidentes de tránsitoPrevención y control, Ginebra-Suiza, Abril. 2001.
3. Maldonado G, Monagas A, Rivero D, Ruotolo A, Urbaneja D, D`Suze C, et al. Accidentes de tránsito ocurridos entre el kilómetro 8 y 50 de la autopista Centro-Occidental Lara-Zulia durante el año 2007. Boletín ANM. 2010;2(18).

4. Peden M, Scurfield R, Sleet D, Mohan D, Hyder A, Jarawan E, et al. World report on road traffic injury prevention. In: Organization WH, editor. Geneva; 2004.

5. Hazen A, Ehiri JE. Road Traffic Injuries: Hidden Epidemic in Less Developed Countries. J Natl Med Assoc. 2006;98(1):73-82.

6. Choquehuanca-Vilca V, Cárdenas-García F, CollazosCarhuay J, Mendoza-Valladolid W. Perfil epidemiológico de los accidentes de tránsito en el Perú, 2005-2009. Rev Perú. Med Exp Salud Pública. 2010;27(2):162-69.

7. Prada R, Río MC del, Álvarez FJ. Accidentes de tráfico en la población Española. Med Clin. 1995;105:601-604.

8. Hyder AA. Road safety is no accident: a call for global action. Bull World Health Organ. 2004;82(4):240.

9. Nantulya VM, Reich MR. The neglected epidemic: road traffic injuries in developing countries. BMJ. 2002;324(7346):1139-41.

10. Nantulya VM, Sleet DA, Reich MR. The global challenge of road traffic injuries: Can we achieve equity in safety? Inj Control Saf Promot. 2003;10:3-7.

11. Patton GC, Coffey C, Sawyer SM, Viner RM, Haller DM, Bose K, et al. Global patterns of mortality in young people: a systematic analysis of population health data. Lancet. 2009;374(9693):881-92.

12. Murray CJ, Lopez AD. Mortality by cause for eight regions of the world: Global Burden of Disease Study. Lancet. 1997; 349(9061):1269-76.

13. García H, Vera C, Zuluaga L, Gallego Y. Caracterización de personas lesionadas en accidentes de tránsito ocurridos en Medellín y atendidas en un hospital de tercer nivel, 1999-2008. Rev. Fac. Nac. Salud Pública 2010;28(2):105-117.

14. Martínez LJF. Muertes y lesiones por accidente de tránsito en Colombia: 2007. Forensis. 2008:221-4.

15. Grisales Y, Robayo M. Análisis de la percepción de los motociclistas sobre la reglamentación vial y su relación con la accidentalidad en Medellín. Escuela de Ingeniería de Antioquia. 2013.

16. Hincapié C, Peláez L, Pérez J, Ramírez W. Traumatismo en accidentes de motociclistas atendidos por el cuerpo oficial de bomberos Medellín en 2008. (Estudio presentado como requisito para optar al título de tecnólogo en atención pre hospitalaria). Facultad de Medicina. Tecnología en atención pre hospitalaria. Universidad CES Medellín. 2009;56.

17. Cárdenas E. Diagnóstico sobre la accidentalidad de motociclistas en Medellín entre enero de 2004 y marzo de 2007. Rev Salud Pública de Medellín. 2007;2(1):7181.

18. Fondo de Prevención Vial. Boletín de prensa: Poca preparación de motociclistas, causa aumento de accidentalidad. Bogotá, Septiembre 8 de 2006. (Datos del Instituto de Medicina Legal y Ciencias Forenses). 
19. Comité de Ensambladores de Motocicletas Japonesas. Tercer estudio socio-demográfico de usuarios de motos en Colombia. 2008.

20. Vásquez RA. Causas de los accidentes de tránsito desde una visión de la medicina social. El binomio alcoholtránsito. 2004;20:178-186.

21. Secretaría de Transporte y Tránsito de Medellín. Análisis técnico de sitios con mayor accidentalidad en el año 2009. 2010: 19.

22. World Health Organization. 10 facts about road safety: fact file. Geneva: WHO; 2009.

23. Organización Mundial de la Salud Departamento de Prevención de la Violencia y los Traumatismos y Discapacidad (VIP). Informe sobre la situación mundial de la seguridad vial 2009. Es hora de pasar a la acción 2009: 227.

24. World Health Organization. Global status report on road safety: time for action. Geneva: WHO; 2009.

25. Bernada M, Alessandrini D, Gallego R, et al. Lesiones graves y fatales en niños y adolescentes uruguayos secundarias a siniestros de tránsito por motos. Arch. Pediatr. Urug. 2013;84(2).

26. Aristizábal D, Gonzales G, Suárez J, Roldán P. Factores asociados al trauma fatal en motociclistas en Medellín, 2005-2008. Revista Biomédica. 2012;32(1).

27. Sánchez E. Hacia un análisis sociológico de la siniestrabilidad vial. Universidad de A Coruña. Revista de Ciencias Sociales. 2012;52.

28. Corporación Fondo de Prevención Vial. Estudio análisis de alternativas y diseño funcional de una motovía en la ciudad de Cali. 2008.

29. Stuckler D, Basu S, Suhrcke M, Coutts A, McKee M. The public health effect of economic crises and alternative policy responses in Europe: an empirical analysis. The Lancet. 2009;374(9686):315-32.

30. Bernada M. Niños con lesiones graves por siniestros en moto. Un problema de salud emergente. Arch Pediatr Urug. 2012;83(2): 117-120. 